Check for updates

The BMJ

Cite this as: BMJ 2022;376:0132 http://dx.doi.org/10.1136/bmj.0132 Published: 19 January 2022

\section{Covid-19: Lateral flow tests in children fail minimum performance standards, study finds}

\section{Elisabeth Mahase}

Many lateral flow antigen tests commonly used to detect covid-19 in children fall short of the minimum standards set by the World Health Organization, as well as UK and US regulators, researchers have said. ${ }^{1}$

The research team from the University of Manchester and Germany's Institute for Quality and Efficiency in Health Care analysed 17 studies (12 peer reviewed and five preprints) published in English between January 2020 and May 2021. These involved 6355 children who used eight antigen tests from six different brands. The team compared the accuracy of lateral flow tests with polymerase chain reaction (PCR) tests.

The paper, published in BMJ Evidence-Based Medicine, found that the overall sensitivity of the evaluated tests was just over $64 \%$ and the overall specificity was just over $99 \%$.

It said that no test "fully satisfied the minimum performance requirements" as recommended by WHO (sensitivity of at least $80 \%$ and specificity of at least $97 \%$ ), the US Food and Drug Administration (80\% sensitivity), or the UK's Medicines and Healthcare Products Regulatory Agency (80\% with two sided 95\% confidence interval entirely above $70 \%$ and minimum acceptable specificity of $95 \%$ with two sided $95 \% \mathrm{CI}$ entirely above $90 \%$ ).

The researchers said that the findings cast doubt on the effectiveness of lateral flow test use in schools. In the UK, lateral flow tests are recommended by the government for people without covid-19 symptoms, while those with symptoms are advised to get a PCR test.

Data from the 17 included studies were pooled to measure diagnostic sensitivity and specificity. In 11 of the test evaluations, samples were collected from the throat and nose, while in the rest samples were collected only from the nose.

Among children with symptoms, the pooled diagnostic sensitivity was just under $72 \%$ and the pooled diagnostic specificity was just under $99 \%$, based on 3413 children in 13 studies. Among children without symptoms the pooled diagnostic sensitivity was just over $56 \%$ and the pooled diagnostic specificity was just over $98.5 \%$, based on 2439 children in 10 studies.

The team said the differences in test performance between children with and without symptoms shows that the sensitivity and specificity are not inherent test characteristics. They also highlighted that in all but one study, testing was conducted by trained staff. As such, they could not assess sample collection by untrained people or self-testing, which they said would likely worsen performance.

Other study limitations included that the review was restricted to eight tests, despite there being more than 500 on the market. Additionally, the researchers noted that all included studies were performed before covid-19 vaccines were rolled out to children and that most people identified as PCR positive were likely infected with wild type SARS-CoV-2.

1 Fujita-Rohwerder N, Beckmann L, Zens Y, et al. Diagnostic accuracy of rapid point-of-care tests for diagnosis of current SARS-CoV-2 infections in children: a systematic review and meta-analysis. BMJ Evid Based Med 2022. doi: 10.1136/bmjebm-2021-111828. https://ebm.bmj.com/doi/10.1136/bmjebm-2021-111828

This article is made freely available for personal use in accordance with BMJ's website terms and conditions for the duration of the covid-19 pandemic or until otherwise determined by BMJ. You may download and print the article for any lawful, non-commercial purpose (including text and data mining) provided that all copyright notices and trade marks are retained. 